\title{
Monseigneur Bourget et la reconstruction de la cathédrale de Montréal (suite)
}

\section{Léon Pouliot}

Volume 17, numéro 4, mars 1964

URI : https://id.erudit.org/iderudit/302310ar

DOI : https://doi.org/10.7202/302310ar

Aller au sommaire du numéro

Éditeur(s)

Institut d'histoire de l'Amérique française

ISSN

0035-2357 (imprimé)

1492-1383 (numérique)

Découvrir la revue

Citer cet article

Pouliot, L. (1964). Monseigneur Bourget et la reconstruction de la cathédrale de Montréal (suite). Revue d'histoire de l'Amérique française, 17(4), 471-489.

https://doi.org/10.7202/302310ar d'utilisation que vous pouvez consulter en ligne.

https://apropos.erudit.org/fr/usagers/politique-dutilisation/ 


\section{MONSEIGNEUR BOURGET ET LA RECONSTRUCTION DE LA CATHÉDRALE DE MONTRÉAL*}

(suite)

\section{LA REACTION DU DIOCÉSE}

La réaction du diocèse au mandement du 19 mars en fut une de consternation: émotion universelle et profonde. L'unanimité se fit d'emblée sur un point: nécessité d'empêcher l'évêque d'aller mendier à travers le monde pour les besoins de Montréal. Mais sur les moyens d'arriver à cette fin, il y avait division dans les esprits. Indifférentes quant au site de la future cathédrale et profondément attachées à la personne de l'évêque, les paroisses rurales font preuve, dès la première heure, d'une belle générosité. Entre tant d'exemples, il suffira d'en retenir un, que nous empruntons à $L a$ Minerve du 9 avril:

Dans la paroisse de la Longue-Pointe, dont tous les habitants fondaient en larmes, dimanche dernier, en entendant la lecture du mandement de Mgr de Montréal, on s'est mis en œuvre pour répondre à l'appel qui vient d'être fait à tout le diocèse pour la reconstruction de la cathédrale et de l'évêché.

Et déjà dans cette paroisse de 60 familles, on a recueilli près de $£ 50$.

Le personnel de l'évêché sort enfin de sa réserve. La Minerve du 9 avril commente favorablement une brochure qui se répand dans le public, et dont l'auteur serait le chanoine P.-A. Pinsonneault, futur évêque de Sandwich ${ }^{1}$. L'Appel aux Catholiques ${ }^{2}$

* Voir notre Revue, XVII : 340-362.

1 Poulin, Mémoires, 184.

2 Appel aux catholiques du diocèse de Montréal, pour la reconstruction de la cathédrale, détruite par l'incendie du huit juillet 1852. La brochure de 24 pages est reproduite dans Mand. des évêques de Montréal, VIII: 103116. 
cite les passages essentiels du mandement du 19 mars et l'accompagne des réflexions suivantes:

Quel est le catholique qui, après la lecture de cette lettre si touchante, ne se sentira pas profondément attristé de voir son Vénérable Evêque qui, depuis treize ans qu'il gouverne ce diocèse, s'est consumé de travaux la nuit comme le jour pour le bonheur de son troupeau, obligé d'aller à l'étranger solliciter des secours que Sa Grandeur hésite à demander ici, et que tout le monde sait que nous pouvons trouver en abondance dans le diocèse de Montréal; ce serait assurément une honte pour cette riche partie du pays de souffrir qu'on aille ailleurs solliciter les sommes nécessaires pour bâtir convenablement l'évêque d'une ville et d'un diocèse comme Montréal, où l'on voit tous les jours arriver, même des vieux pays de l'Europe, des évêques, des religieux, des missionnaires, qui viennent implorer la charité publique en faveur de leurs établissements et s'en retournent en bénissant Dieu des abondantes aumônes qu'ils ont recueillies dans ce petit coin de notre Amérique ${ }^{3}$.

L'appel aux Catholiques cite de très larges extraits de la Pastorale sur le diocèse de Saint-Hyacinthe, dont nous avons déjà parlé. Il insiste sur la facilité de réaliser le projet de $\mathrm{Mgr}$ Bourget:

Le diocèse de Montréal se compose de plus de cent paroisses. Supposons que dans cent paroisses, les unes dans les autres, il se trouve deux cents personnes (et dans la plupart il s'en trouve un plus grand nombre) capables de donner une piastre par année ou vingt mille piastres par année, et au bout de quatre ans nous aurons réalisé la magnifique somme de vingt mille louis. Dans la ville de Montréal, si on se donne le trouble de bien organiser toutes choses, on n'aura pas de peine sur plus de trente-cinq mille personnes ${ }^{4}$, à en trouver dix mille qui donneront volontiers une piastre par année, pendant qua-

3 Mand., VIII : 109-110.

4 Une statistique, publiée dans le True Witness du 9 avril 1852, fixait à 41,464 le nombre des catholiques de Montréal. Il y avait 16,170 protestants et 181 Juifs. Population totale de la ville: 57,815 . 
tre ans, et ainsi nous nous trouverons avec une somme de trente mille louis pour former un établissement digne du culte de la religion catholique à Montréal ${ }^{5}$.

L'auteur se tait prudemment sur le site de la future cathédrale. Mais il conclut que s'il y a entente et bonne volonté le succès est assuré ${ }^{6}$.

L'Appel aux Catholiques avait pour but de créer un mouvement de sympathie à l'évêque en faisant une large publicité à des textes importants que les fidèles n'avaient entendus que du haut de la chaire, et cela dans une atmosphère d'émotion intense, comme nous l'avons dit. Il s'adressait surtout à la ville de Montréal. A-t-il produit son effet ?

Il était connu du public, il avait été accueilli favorablement par la presse, quand Montréal enfin démarre. Le 12 avril avait lieu dans les salles de l'Institut National ${ }^{7}$, sous la présidence du juge Mondelet, une assemblée publique. On y arrêtait des résolutions et l'on chargeait un comité de rédiger à l'adresse de $\mathrm{Mgr}$ Bourget une requête conforme aux dites résolutions ${ }^{8}$. On communiqua le texte de celle-ci à l'évêque et comme il manifeste le désir de répondre en public, on convoqua pour le dimanche, 17 avril, une grande assemblée qui se tiendrait "près des ruines de la cathédrale". Il y eut d'abord ce qu'on appelle "les procédés de l'assemblée": communication à la foule, quelque 5000 ou 6000 personnes, des résolutions de la réunion du 12 avril, opposition énergique et unanime au projet d'aller mendier à l'étranger. Puis, une délégation composée de MM. Jacques Viger, Côme-Séraphin Cherrier et A. Delisle, alla prévenir l'évêque que l'heure était venue pour lui de recevoir l'adresse des citoyens et d'y répondre. C'était au juge Mondelet qu'il appartenait de recevoir Mgr Bourget et de lire le texte de l'adresse au prélat. Il le fit de bonne grâce.

5 Mand., VIII : 115 .

6 Ibid., 116.

7 Sur l'Institut National élevé en opposition à l'Institut Canadien, voir la courte note que nous avons donnée à la RHAF, XIV (mars 1961): 481-486.

${ }^{8}$ La Minerve, 14 avril 1853. 
Ensuite Montréal vit ce qu'il n'avait jamais vu: Mgr Bourget lisant un texte rédigé d'avance et ne se permettant pas d'y ajouter un mot de commentaire. Il crut bon de s'expliquer. Écoutons-le:

C'est contre mon ordinaire, comme vous le savez; car, quand je parle à mes enfants, j'aime beaucoup mieux laisser mon cœur à ses impressions du moment que de laisser courir une plume qui, quelle que soit son exactitude, ne peut peindre qu'imparfaitement ce que sent un cœur navré de douleur, quand il rencontre des cœurs qui compatissent à ses malheurs, comme j'en rencontre aujourd'hui des milliers. D'ailleurs, je crains que ma faible voix ne puisse porter à toutes les oreilles ici ouvertes pour m'entendre les impressions de mon âme. Je m'impose done l'obligation de vous lire ces quelques lignes que j'ai tracées à la hâte sur ce papier pour qu'elles soient publiées, et puissent par là aller à la connaissance de tous. Je dois parler franchement et ouvertement, c'est mon devoir, et c'est aussi ma pensée ${ }^{9}$.

L'évêque rappelle les liens qui attachent son cœur au quartier Saint-Jacques, il rappelle le souvenir de son maître, Mgr Lartigue. "Pas un dans cette ville, dit-il, ne peut aimer autant que moi l'emplacement qui nous réunit aujourd'hui, et je pense que vous me croirez sur parole." Il déclare ici pour la première fois, - car il ne peut rien cacher à ses enfants, - que l'incendie de la cathédrale lui est apparue comme une occasion providentielle de faire de l'établissement de l'évêque le premier établissement de la ville et même du diocèse. "Du moins, me regardé-je comme obligé d'y travailler de toutes mes forces."

Je vois cependant, messieurs, que le but de votre adresse est de relever les ruines de l'ancienne cathédrale et de l'évêché, et j'en conclus que ce serait votre intention de fixer pour toujours la résidence de l'évêque sur l'emplacement actuel. Or, si ce projet était mis à exécution, l'occasion qui se présente de donner à l'établissement de l'évêque l'importance qu'il doit avoir, c'est un coup manqué pour toujours

9 Mand., VIII : 99. 
car, si aujourd'hui des intérêts particuliers empêchent la translation projetée, ce sera beaucoup plus difficile de l'effectuer dans un temps qui peut être plus ou moins reculé. Dans ce cas, toujours la cathédrale se verra entourée, dans cette ville, d'églises plus augustes par leur magnificence. Je dois ajouter que bon nombre d'églises dans nos fertiles campagnes seront plus belles et plus majestueuses. Ce que vos yeux peuvent apercevoir de tous côtés prouve ce que je dis; et dans quelques années, lorsque de nouvelles églises auront surgi sur tous les points de notre cité, on le verra encore plus clairement. Maintenant, est-ce la pensée de la ville de faire à son évêque une église qui devra le faire rougir, comme rougissait de la sienne l'évêque de Marseille, avec qui j'ai le bonheur d'être intimement uni et qui me disait, lorsque je passai par cette ville, qu'il invitait les étrangers à ne pas visiter sa cathédrale ${ }^{10}$.

D'après le compte rendu, la majorité était favorable à l'ancien site, mais parut "recevoir cette décision de Monseigneur avec beaucoup de respect, si on excepte cependant quelques expressions de mécontentement échappées à des personnes qui ne savent pas assez bien se contenir quand leurs désirs sont froissés" ${ }^{11}$.

Ainsi, cette grande assemblée du 17 avril aboutissait à un échec. La Minerve du 21 constate que le désappointement de la ville est immense. Elle reconnaît et le droit de l'évêque et l'attachement des fidèles au quartier Saint-Jacques; elle fait mieux et cherche une solution: "Des personnes ont compris que Mgr refusait de laisser rebâtir l'église Saint-Jacques sur le terrain consacré où sont maintenant ses ruines. Nous ne comprenons pas ainsi sa réponse: il refuse d'y fixer de nouveau sa cathédrale et son évêché. Mais il n'a pas dit qu'il refusait d'y voir une église pour le service des environs."

Le lendemain, le comité des citoyens s'engageait dans cette voie. Il demandait à l'évêque la permission d'ériger sur le site de la cathédrale une église de quartier, moyennant quoi la sous-

10 Ibid., 101-102.

11 Ibid. 
cription en faveur du projet du Coteau Baron serait lancée immédiatement.

Mgr Bourget accorde volontiers la permission: "J'observe toutefois que l'on doit, avant tout, se mettre en mesure de prélever les souscriptions de la ville, et si elles sont ce qu'elles promettent d'être, nous nous mettrons ensemble à l'œuvre, et il faut l'espérer, nous la ferons avec bonheur, parce que nous la ferons en prix ${ }^{12}$." Mais il tient à expliquer son attitude relativement au site de la future cathédrale.

Je ne vous dirai pas ce qu'il en a coûté à mon cœur, de contrister, comme je l'ai fait, le vœu populaire. Je vous ferai seulement observer que si j'ai dans cette ville quelque popularité, je l'ai toute risquée dans une occasion si solennelle, et qui pouvait être si décisive pour moi.

Et en effet, je me suis exposé au grave inconvénient d'avoir contre moi la majorité des citoyens, celle de mes bons voisins et de mes meilleurs amis. Je me suis résigné à tout, parce que mon cœur me disait que je ne voulais que le plus grand bien, et parce que j'avais l'intime conviction que plus tard je serais compris ${ }^{13}$.

Admirable attachement de l'évêque à ce qu'il considère comme son devoir !

Le 4 juin, on apprenait qu'en vertu d'une entente intervenue entre Mgr Bourget et le Séminaire de Saint-Sulpice, celui-ci prenait à sa charge la reconstruction de l'église Saint-Jacques, qui servirait de cathédrale en attendant des temps meilleurs ${ }^{14}$. La voie était ouverte au vœu de Mgr Bourget. Mais voici que surgit un autre problème: un projet de chemin de fer MontréalOttawa rend aléatoire la cession du Coteau Baron à la corporation épiscopale ${ }^{15}$. Et l'on voit apparaître l'ouest de la ville comme

12 La Minerve, 3 mai: Mgr Bourget à MM. C.-S. Cherrier, Joseph Grenier et T.-J.-J. Loranger.

13 Ibid.

14 La Minerve, 4 juin.

15 C'est la raison apportée par M. Poulin, dans ses Mémoires, op. cit., 184. De son côté, Mgr Bourget écrira: "Si d'abord, nous avons eu le projet de transporter notre établissement à quelques arpents du site de la cathé- 
site possible de la future cathédrale. Ainsi, lit-on dans $L a$ Minerve: il y aura trois classes de souscripteurs: ceux qui donnent sans indication de site, ceux qui préfèrent le Coteau Baron et ceux qui préfèrent l'ouest de la ville. "Les souscripteurs de la seconde et troisième classes devront attacher à leur souscription l'alternative d'une souscription moindre au cas d'une décision contraire à leurs désirs." Disposition malheureuse: elle offrait aux mécontents, car il y en a toujours, une occasion de concilier leur mauvaise humeur avec un certain air de générosité.

Quoi qu'il en soit, le comité va de l'avant; il fait imprimer des blancs de souscription, nomme pour chaque quartier de la ville des solliciteurs qui iront de maison en maison recueillir les dons et les promesses de dons. Les obstacles d'hier paraissent vaincus et on se laisse aller à l'espérance.

Mgr Bourget n'avait pas attendu la suite de ces événements pour renoncer au projet de voyage en Europe. Le 21 avril, il écrit: "Les démonstrations de la ville m'obligent à retarder mon départ pour l'Europe. Peut-être y renoncerai-je tout à fait. Cela dépendra des événements maintenant pendants dans notre cité ${ }^{16}$." Dans le Mandement de visite, qui est du 16 mai, il déclare que, cédant aux représentations du clergé et des laïcs, il a différé son voyage en Europe ${ }^{17}$.

Hélas ! trois fois hélas ! tant de réunions, de belles paroles, de promesses, d'espérances n'ont rien produit! Témoin le communiqué que publiait La Minerve du 30 décembre 1853:

Evêché de Montréal. Nous sommes priés d'annoncer que Mgr de Montréal ne se trouvant pas plus convenablement logé que l'année dernière, sera privé du plaisir de recevoir les visites d'usage, au premier de l'an. Cette condition de gêne où se trouve $\mathrm{Mgr}$

\footnotetext{
drale incendiée, c'est qu'alors tout semblait promettre que les grandes améliorations en contemplation se feraient dans le quartier. Mais Nous y avons renoncé dès que les travaux ont été ouverts dans la partie supérieure de la ville." Mand., II : 487. Berthier.

${ }^{16} A A M$, Lettres de $M g r$ Bourget, 8, 20. A M. Gagnon, curé de 17 Mand., 2 : 358-366.
} 
de Montréal, deux ans après l'incendie désastreux qui a consumé son église et sa résidence, nous fournit l'occasion de rappeler aux messieurs qui composent le comité chargé de prendre les moyens de venir en aide à Sa Grandeur pour reconstruire son établissement épiscopal, qu'ils n'ont pas encore toutà-fait achevé leur tâche. Ils ont mis des livres en circulation, mais à l'heure qu'il est, ils n'ont pas encore obtenu, ni même demandé, pensons-nous, les rapports des collecteurs. Ne serait-il pas à propos de voir le montant des secours sur lesquels Mgr peut compter pour se rétablir convenablement?

Comme il en avait été l'année précédente, la reconstruction de la cathédrale n'est que l'un des soucis qui sollicite l'attention de Mgr Bourget en 1853. Chaque jour lui apporte des problèmes; il y porte toute son attention. A quoi il faut ajouter le zèle de Dieu et des âmes qui ne le quittait pas un instant et qu'il s'efforçait de communiquer aux autres.

A l'occasion du nouvel an, il avait l'habitude de proposer aux communautés religieuses une grande intention apostolique. Cela ne comportait aucune prière spéciale, aucun exercice de piété nouveau: c'était un moyen d'empêcher l'âme de s'enliser dans la routine, en la faisant participer aux grands intérêts de l'Église universelle. Le 1er janvier 1853, il écrit aux religieuses de l'Hôtel-Dieu:

Cette année, vous allez vous faire victimes pour nos frères séparés. Hélas! il s'en trouve dans ce diocèse et dans celui de St. Hyacinthe, qui n'en font qu'un, au moins cent mille. Voilà donc cent mille âmes qui se perdent autour de nous. Cette perte est pour tout cœur religieux une plaie toujours saignante. Aussi, allez-vous vous immoler toute l'année, pour obtenir le retour au bercail de tant d'âmes égarées ... ${ }^{18}$.

Il recommande la même intention au clergé, et ici comme ailleurs, il paie d'exemple, il tient à faire sa part:

${ }^{18}$ AAM, Lettres de Mgr Bourget, 7, 560-563. 
Voulant, comme il est juste, Nous mettre à contribution, pour une bonne part du travail qu'il va devenir nécessaire de nous imposer pour ramener à la vraie foi nos frères séparés, nous allons, pour quelque temps, faire diversion avec les affaires courantes, afin d'aller étudier l'anglais dans une des missions de ce diocèse ${ }^{19}$.

Depuis 1844, date d'érection de la province ecclésiastique, Mgr Bourget désirait ardemment la tenue d'un concile. Il n'a rien négligé pour souligner l'importance du premier concile provincial de Québec (1851). Son coadjuteur, Mgr Jean-Charles Prince, et un prêtre de l'évêché, M. Joseph Larocque, furent chargés d'aller soumettre les décrets à l'approbation de Rome. Celle-ci obtenue, il restait à promulguer les décisions conciliaires. Le 17 novembre 1852, Mgr Bourget écrivait à Mgr Prince: "J'ai commencé à rédiger une Lettre Pastorale pour publier les décrets du concile de Québec, et je passe plusieurs jours de suite sans pouvoir écrire une seule ligne ${ }^{20}$.

La Pastorale au clergé, longue de 20 pages, est du 1er janvier 1853. L'évêque décrit avec onction les événements auxquels il a participé, il dit et l'esprit qui animait les Pères et l'esprit avec lequel les décrets doivent être reçus et observés ${ }^{21}$.

Le 10 février, il se réjouit de la fondation de l'Université Laval. Il écrit à Mgr Turgeon: "Rien ne m'est plus agréable que la bonne nouvelle que m'apprend V.G. de l'heureuse issue des démarches entreprises pour l'érection d'une Université dans le Séminaire de Québec, V.G. peut croire que j'y concours de toutes mes forces ${ }^{22}$.

Le 1er avril, il adresse un mot de félicitations au Dr Meilleur, surintendant de l'instruction publique, au sujet du Guide de l'Instituteur:

Pour l'amour que je porte aux intérêts de la jeunesse, je dois vous exprimer ma satisfaction, surtout pour

${ }^{19}$ Mand., 2: 339. Le séjour de Mgr Bourget à Saint-Colomban a duré du 20 janvier au début d'avril.

20 AAM, Lettres de Mgr Bourget, 7, 483-485.

21 Mand., II: 319-340.

22 AAM, Lettres de Mgr Bourget, 7, 585. 
la dernière partie de ce document qui commence par ces mots: "Les professions libérales sont généralement plus que remplies, etc ..." Si les idées qui sont exprimées dans tout ce passage étaient universellement adoptées, que de maux cesseraient de menacer notre pays par la restriction dans de justes limites de la haute éducation collégiale ${ }^{23}$.

En 1853, Mgr Bourget voulut faire personnellement la visite de toutes les paroisses du diocèse. Il y consacra intégralement les mois de juin, juillet et août, ainsi que la première quinzaine d'octobre. La mission, c'est-à-dire la prédication, qui était jusqu'ici un élément de la Visite Pastorale, est confiée aux Pères Oblats qui la feront en un autre temps ${ }^{24}$. En se limitant à l'essentiel, confirmation des enfants, visite du cimetière, des fonts baptismaux et autres objets du culte, l'évêque peut consacrer une journée à chaque paroisse ${ }^{25}$. Le 22 juin, il est à Saint-Jacques l'Achigan, et il décide d'y transférer la maison mère des Sœurs de Sainte-Anne, qui manquait d'espace vital à Vaudreuil ${ }^{26}$.

Et c'est pendant la visite de Saint-Cyprien qu'il donne à la communauté un chapelain dans la personne de M. L.-A.-D. Maréchal ${ }^{27}$. Ces deux faits ne sont pas sans importance dans notre histoire religieuse.

${ }^{23} \mathrm{~A} A M$, Lettres de Mgr Bourget, 8, 5. - Par haute éducation collégiale, Mgr Bourget entend le cours classique. Comme tous les bons esprits de son époque, il souhaitait pour la jeunesse une éducation plus diversifiée. Or, entre l'enseignement primaire et le cours classique, il n'y avait pratiquement rien. De là un nombre croissant de jeunes gens instruits, mais incapables de faire carrière dans les professions libérales, déjà encombrées; et de plus ils n'avaient aucune préparation à l'industrie, au commerce, à l'agriculture. Ces jeunes désouvrés devenaient vite des mécontents et n'étaient sans exercer quelque influence sur le peuple. Car ils avaient appris au Collège l'art d'écrire et de parler en public. Mgr Bourget souhaitait, entre l'enseignement primaire et le cours classique, une éducation moyenne, sans latin et sans grec, qui eût permis aux jeunes que les professions libérales ne pouvaient absorber, de gagner honnêtement leur vie et de tenir un rang honorable dans la société.

24 Mand., II: 358-366.

25 AAM, Pièces et Actes, VIII: fol. 87V.: "Itinéraire de la visite Pastorale pour 1853".

26 Annales Religieuses et Historiques de St-Jacques l'Achigan, 48: "Le 22 juin 1853 fait une nouvelle visite dans la paroisse St-Jacques, ce fut à cette occasion qu'il régla une question de la plus haute importance." est du 15 .

$27 \mathrm{Mgr}$ Bourget était à St-Cyprien, le 13 août. La lettre de nomination 
Mgr Bourget n'était donc pas à Montréal lors des échauffourées sanglantes et mortelles suscitées à Montréal, le 9 juin, par les prédications du moine apostat Gavazzi ${ }^{28}$. Mais il accueille chaleureusement Mgr Gaetano Bedini, en route vers sa nonciature du Brésil, et qu'une vague de fanatisme déferlant alors sur les États-Unis invite à chercher refuge au Canada ${ }^{29}$.

Le 27 décembre, Mgr Bourget adressait au diocèse une Pastorale, à l'occasion de la nouvelle année. Il y traitait d'un sujet alors de grande actualité, tant en Europe qu'en Amérique: les tables tournantes; ce document nous révèle un aspect de l'âme de Mgr Bourget: sa modernité. Loin de bouder les grandes découvertes scientifiques de son époque, il y voyait des dons de Dieu à l'homme et des moyens pour celui-ci de s'élever au créateur: "Quand donc de nouvelles inventions se présentent dans le monde, toutes plus merveilleuses les unes que les autres, Nous nous contentons de les admirer et d'en bénir la divine Providence, en disant avec le Saint Roi: Que vos ouvres, Seigneur, sont magnifiques! Ainsi sommes-nous sans cesse dans l'admiration, en contemplant la puissance de la vapeur qui, sur terre et sur mer, traîne à sa suite, avec la rapidité du vent des masses énormes qui apparaissent aux yeux étonnés comme des montagnes ambulantes et flottantes. Plus encore sommes-nous ravi de la vitesse de l'électricité qui, comme l'éclair, va porter notre pensée à des milliers de lieues (-..--).

C'est ainsi, N.T.C.F., que nous devons tous considérer les œuvres de notre Dieu, dans l'ordre naturel, pour tout rapporter à sa gloire. C'est là la grande leçon de la religion, qui apprend à l'homme à s'élever, jour et nuit vers son Créateur, pour le louer et le bénir de tant de biens qu'il en reçoit chaque jour. Elle lui apprend en même temps à user de ces biens avec humilité, et à ne pas franchir les bornes qu'il a tracées aux opérations de

28 Voir sur ce sujet l'excellent ouvrage de Robert Sylvain: "Clerc, Garibaldien, Prédicant des Deux Mondes, Alessandro Gavazzi (1809-1889) (Québec, 1962), I-II.

29 AAM, Lettres de Mgr Bourget, 8, 119. Au Cardinal Barnabo, 12 sept. 1853. Cette visite écrit l'évếque, est un trait de la Providence, après l'affaire Gavazzi. Elle a contribué à enraciner dans les âmes le culte du Pape. 
l'esprit humain, aussi bien qu'aux flots de la Mer. Huc usque venies, autrement, l'on tombe dans de pitoyables erreurs, d'autant plus dangereuses qu'elles sont moins tangibles.

D'après ces principes, les Tables Tournantes, considérées comme choses purement naturelles, et vues de l'œil humain, n'offraient aucun danger. Mais malheureusement, on s'en est écarté, et déjà on a à déplorer de funestes conséquences. Car on a imprudemment dépassé les barrières que Dieu lui-même a fixées pour mettre un frein à l'orgueil et à la vanité de l'homme, on a voulu faire parler des tables sans intelligence, pour savoir ce qui se passe dans le royaume des esprits. Voilà l'abus que Nous voulons signaler à votre attention, dans la ferme confiance que, le connaissant, vous l'éviterez ${ }^{30}$."

Après avoir établi que les tables tournantes relèvent de la superstition et non de la foi, il en décrit les effets déplorables. Par son abondante documentation et par sa solidité: cette Pastorale fut remarquée non seulement au Canada, mais aussi en France.

\section{LE SITE DE LA CATHÉDRALE}

Jusqu'ici nous savons que la cathédrale ne sera pas reconstruite dans le quartier Saint-Jacques. Pour des raisons indépendantes de la volonté de Mgr Bourget, il fallut renoncer au Coteau Baron. Mais où sera-t-elle établie ?

Pour comprendre le geste que Mgr Bourget posera bientôt, il faut se rappeler le souci qu'il avait de multiplier dans la ville les endroits de culte: souci pastoral inspiré par le rapide développement de la ville. Au fur et à mesure que Montréal s'affirmait comme ville commerciale et industrielle, la population grandissait; elle eût tôt fait de déborder ce que nous appelons aujourd'hui "le vieux Montréal". Celui-ci se prolonge bientôt aux quatre points cardinaux en des agglomérations humaines, en des villages homogènes, c'est-à-dire dont les habitants étaient de langue française et de foi catholique.

${ }^{30}$ Mand., II : 388-389. 
En vertu de privilèges qu'il avait reçus de Rome et que Mgr Bourget avait lui-même reconnus en 1843, le Séminaire de SaintSulpice était le seul curé et le curé perpétuel de la ville. C'était à lui qu'il appartenait de pourvoir au bien spirituel de Montréal, et donc d'ériger des églises et des maisons d'enseignement dans les limites de sa juridiction. Et il le fit. Mais devant la rapide progression numérique des habitants et leur dispersion dans l'espace, ce devoir devenait extrêmement onéreux, voire irréalisable. Le Séminaire multiplia les dessertes, érigea dans chaque agglomération un édifice qui servait d'école, et où le dimanche on célébrait la messe pour la commodité des fidèles. Ceux-ci étaient visités, assistés en cas de maladie par un vicaire désigné, mais qui habitait la Paroisse. Baptêmes, mariages, sépultures avaient lieu à Notre-Dame, ce qui n'allait pas sans inconvénients en un temps où les moyens de transport étaient rares et dispendieux et où les rues étaient mal entretenues. Dès 1846, Mgr Bourget voulut ériger ces localités en paroisses avec curés résidants. Il patienta 20 ans avant de voir reconnu par Rome son droit d'évêque et il patienta encore dix ans avant de triompher des obstacles de l'autorité civile.

Mgr Bourget, avons-nous dit, avait le souci pastoral de multiplier les endroits de culte, afin de faciliter aux fidèles la pratique religieuse. De plus, dans la conception qu'il avait de l'Église, celle-ci devait être présente, et d'une présence honorable là où se manifestaient la grandeur, la puissance et la richesse de ce monde. C'est parce que Kingston était devenue la capitale des Canadas-Unis qu'en 1841 il y avait dirigé des religieuses enseignantes - la Congrégation de Notre-Dame - et 4 ans plus tard les Sœurs de l'Hôtel-Dieu. Dans ce milieu protestant, fréquenté par les députés et les ministres des deux provinces, ces deux fondations attesteraient que l'Église a le souci d'instruire chrétiennement la jeunesse et qu'elle entoure de ses soins maternels les pauvres et les malades.

Or, dans le Montréal de 1854, la partie ouest de la ville, le quartier Saint-Antoine, en particulier, était le centre des affaires. On y voyait de beaux édifices commerciaux, de belles églises protestantes, mais de temples catholiques, point. Il n'en sera pas 
toujours ainsi. Au début de juillet 1854, Mgr Bourget se portait acquéreur du site sur lequel s'élève aujourd'hui la BasiliqueCathédrale. Ce serait d'abord une affirmation et une prise de possession: on y élèverait une modeste église et une résidence pour le personnel de l'évêché.

Le 27 août, l'évêque communiquait la nouvelle au clergé et aux fidèles de la ville ${ }^{31}$. Il y justifiait ainsi le choix du site:

"Nous n'avons donc choisi le site actuel que parce que Nous avons cru qu'il réunissait plus d'avantages religieux. En effet, ce quartier devient le centre de toutes les communications de la province; l'évêque y sera donc plus accessible au clergé et aux fidèles du diocèse ${ }^{32}$.

Tous les mouvements matériels se dirigent de ce côté-là; l'action de l'évêque y sera donc plus nécessaire pour la conservation du dépôt des saines doctrines et le maintien des pratiques religieuses. Toutes les grandeurs du monde vont s'y développer: la Religion y doit donc déployer, avec les splendeurs de son culte, toutes ces forces morales, afin de paralyser, autant que possible, cet esprit de matérialisme qui, en courbant insensiblement l'homme vers la terre, lui fait oublier le ciel.

Ce quartier semble donc requérir impérieusement la présence de l'évêque pour se développer au spirituel comme au temporel; ce qui souffre d'autant moins de difficultés que le quartier que Nous laissons (Saint-Jacques) paraît suffisamment doté d'église et de chapelles pour le service divin et d'institutions religieuses pour les œuvres de charité et d'éducation ${ }^{33}$."

A ces raisons essentielles s'en ajoutait une autre qui, cependant, n'était pas "péremptoire": la proximité du cimetière. Mgr Bourget trouvait belle et consolante la conception, héritée des âges de foi, suivant laquelle l'Église, Mère des Vivants et des Morts, pouvait, d'un seul regard, embrasser les uns et les autres

31 Ibid., 486. Lettre Pastorale de Mgr l'évêque de Montréal, concernant la réédification de son église-cathédrale.

32 Allusion au chemin de fer Le Grand Tronc, récemment inauguré et dont la gare Bonaventure se trouvait toute proche de l'évêché.

33 Ibid., 487-488. 
et perpétuer ainsi, entre les générations qui se succèdent, des liens de reconnaissance et d'amour.

L'évêque annonçait aux fidèles de Montréal qu'il irait à domicile recevoir leurs aumônes. Et il le fit; cependant, lit-on dans un vieux document, il désirait moins pour le moment des dons en argent que des souscriptions. Il savait que la construction de la grande cathédrale n'était pas imminente ${ }^{34}$; mais il ne lui était pas indifférent de savoir sur quels secours il pourrait compter quand l'heure serait venue d'aller de l'avant. Les choses en étaient là quand il dut suspendre ses visites à domicile.

\section{LE VOYAGE EN EUROPE (1854-1856)}

Le 17 octobre 1854, Mgr Turgeon, archevêque de Québec, invitait son suffragant de Montréal à représenter la province ecclésiastique à la définition solennelle du dogme de l'ImmaculéeConception, fixée au 8 décembre. L'archevêque voulait par là reconnaître et récompenser le culte tout filial de $\mathrm{Mgr}$ Bourget envers la Sainte Vierge. Celui-ci accepta et, le 23 octobre, il quittait Montréal; il ne devait y revenir que le 29 juillet 1856 . Nous parlerons ailleurs des autres résultats de ce voyage démesurément long. Demandons-nous pour l'instant en quoi il a affecté la reconstruction de la cathédrale.

Quand, le 23 octobre 1854, Mgr Bourget partait pour Rome, le quartier Saint-Antoine voyait s'élever deux modestes édifices religieux: une résidence pour le personnel de l'évêché et une chapelle publique. Celle-ci était bénite par Mgr Prince, le dimanche, 25 février 1855 :

Ce n'était qu'une chapelle, plus que modeste assurément. Mais elle a donné asile, pendant plus de qua-

34 Le 3 juillet 1854, Mgr Bourget écrivait à M. Billaudele, sup. du Séminaire: "Je crois devoir vous informer que je me propose d'acquérir le terrain voisin du cimetière pour y bâtir plus tard (c'est nous qui soulignons) la cathédrale. Pour le moment, je vais y faire élever un édifice, qui servira à la fois de chapelle et de logement aux prêtres de l'évêché. Je sens le besoin de sortir au plus tôt, de l'état où nous nous trouvons (à l'hospice St-Joseph). AAM, Lettres de Mgr Bourget, 8, 430-432. 
rante ans, aux évêques de Montréal et à leur chapitre, pour les offices religieux, et mérite à ce titre qu'on en fasse mention. C'était un vaisseau rectangulaire, bas, mal éclairé, en briques communes, sans aucun style, sans façade, s'ouvrant par une petite porte de côté, sur la rue Cathédrale. Cette église s'est trouvée écrasée en outre, dans la suite par la grandeur et la richesse des superbes édifices qui bordent maintenant le square Dominion. Elle a été démolie en 1895, et remplacée par l'annexe en briques qui relie l'archevêché à la cathédrale actuelle ${ }^{35}$.

Si modestes que soient ces édifices la corporation épiscopale a dû s'endetter pour les mener à terme. Et puis, les quêtes à domicile ont pris fin avec le départ de Mgr Bourget. Le Comité de reconstruction ne se réunit plus. Un vent de découragement souffle sur le coteau Saint-Antoine. Chacun, suivant la pente de son tempérament, tient l'évêque de Montréal au courant. M. Paré aspire à rejoindre Mgr Bourget, afin de quêter pour la cathédrale: car il n'y a rien à espérer de Montréal (3 déc.). L'économe, M. Plamondon, signale que la dette de l'évêché est de $£ 20,000$, que depuis un mois, il n'a pu payer les intérêts. "Je ne comprends pas, ajoute-t-il, que vous songiez à construire maintenant votre cathédrale (22 oct.)." Mgr Larocque est littéralement découragé. "Nous sommes pauvres comme des rats de grange", écrit-il. Il se sent inégal à la tâche, incapable de faire face au clergé ou aux laïques. Il ne voit de salut que dans le prompt retour de Mgr Bourget: "Il me semble que votre absence prolongée ne nous sera favorable sous aucun rapport. Les administrations comme les régences sont des temps de faiblesse ou d'abus (7 Mai 1855)." Tout autre que Mgr Bourget aurait capitulé et serait revenu dare-dare à Montréal. Lui, il n'y pense même pas. La mauvaise humeur, les récriminations des autres sont impuissantes à le décharger de ce qu'il considère comme un devoir sacré et personnel. Ces courriers alarmants produisent un effet tout autre que celui qu'on avait espéré. L'évêque n'a pas demandé à venir en Europe; mais puisqu'il y est, il se fera mendiant. Il

${ }^{35}$ Le diocèse de Montréal à la fin du dix-neuvième siècle (Montréal, 1900), 14. 
soumet le projet au Saint-Siège ${ }^{36}$ et il va de l'avant. Pour préparer les visites qu'il entreprendra bientôt, il rédige et fait imprimer chez Adrien Leclère, Paris, rue Cassette, une brochure de $\mathbf{4 0}$ pages: Appel à l'Ancienne France pour un secours en faveur de la Nouvelle. A l'aide d'extraits de ses Pastorales, il décrit la triste situation de Montréal depuis le 8 juillet 1852, il dit combien il est grand de collaborer à la construction d'une cathédrale et il conclut:

Il est à croire que, plus tard, cette ville pourra faire pour d'autres ce que l'on aura fait pour elle dans cette déplorable circonstance.

Quoi qu'il en soit, les généreux bienfaiteurs de cette église ne seront point oubliés dans les prières qui s'y feront, après qu'elle aura été relevée de ses ruines, aussi longtemps qu'elle subsistera.

Et comme la bonne entente conclue entre la France et l'Angleterre, à l'occasion de la guerre de Crimée, faisait naître les plus grands espoirs, il ajoute:

Cette future cathédrale attestera de plus à la postérité la plus reculée que la France, en embrassant aujourd'hui de si bon cœur l'Angleterre, sa puissante alliée, a senti à l'instant ses entrailles de mère s'émouvoir au seul souvenir du Canada, qui fut si longtemps l'objet de ses vives et tendres sollicitudes.

La correspondance de Mgr Bourget nous permet de le suivre à travers la France. Partout où il passait, il était considéré comme un saint; mais l'hommage s'adressait à sa personne et s'arrêtait là. Les Français n'étaient pas autrement émus par le problème de Montréal. Les oboles insignifiantes reçues par l'évêque en font foi. Devant les inondations qui dévastèrent la France, au printemps de 1856, Mgr Bourget comprit que ses quêtes seraient vraiment importunes, et il décida de rentrer au pays.

36 Avant de quitter Rome, le 8 juillet 1855, l'évêque avait demandé au Saint-Père la permission de quêter à l'étranger pour sa cathédrale. Arch. de la Prop. Scrit. rif. t. 6. Et le 25 juillet, il écrivait au Cardinal Barnabo: "En arrivant en France, je me suis occupé de la collecte que le St-Père m'a permis de faire pour aider Montréal à relever les ruines de sa cathédrale." Ibid. 
A travers tout cela, le projet de reconstruction progressait, il se précisait.

$\mathrm{Au}$ printemps de 1856, le cher et dévoué secrétaire, M. Joseph-Octave Paré, allait rejoindre Mgr Bourget à Paris ${ }^{37}$. Et, entre autres choses, il se voyait assigner la mission de visiter les grandes cathédrales d'Europe et de décider laquelle servirait de modèle à Montréal. M. Paré eut tôt fait d'arrêter son choix sur Saint-Pierre-de-Rome, pas moins ! et Mgr Bourget, il va sans dire, approuva. Dans le mémoire qu'il adresse à $M$. Paré, il déclare que l'extérieur du temple doit être imposant, mais ne doit pas absorber de gros capitaux. "Il vaut mieux réserver ses ressources pour l'intérieur et toujours dans le but de favoriser l'esprit religieux." Et encore: "Si l'on se décidait à bâtir sur le plan de St-Pierre, il faudrait adopter quelques changements pour que tous ceux qui sont dans la grande nef puissent tout voir. Pour cela, il suffirait de reculer la Confession vers le fond de l'église ... ${ }^{38}$." M. Paré n'était pas sans se rendre compte que la dépense serait considérable et qu'il fallait, dès le point de départ, assurer à l'évêque des revenus substantiels et stables. Il pense, comme l'avait fait le chapitre trois ans plus tôt, à une taxe sur les biens du clergé: taxe qui pèserait plus lourdement sur le Séminaire de Montréal. La réponse de Mgr Bourget mérite d'être retenue, parce qu'elle nous livre les pensées de son âme, au moment où il s'apprête à revenir au pays :

Pour ce qui est de votre projet de mettre le Séminaire à contribution pour la reconstruction de la cathédrale, je dois vous faire observer que c'est une chose si grave et si compromettante que je puis seul m'en mêler, s'il y a nécessité d'en venir là. Mais je suis persuadé que si le clergé doit être forcé de payer la dîme à l'évêque, le Séminaire fera sa bonne part et de bon cœur. Quoi qu'il en soit, vous comprenez que je ne puis prier la Propagande d'intervenir dans cette affaire avant d'avoir fait directement appel à

37 Dans une circulaire au clergé, datée du 16 avril 1856, Mgr Larocque annonçait ainsi le départ de M. Paré: "Deux citoyens bienveillants ayant fourni les fonds nécessaires à son voyage, ce monsieur a pu enfin partir pour Paris, il y a quelques jours." Mand., III : 184.

38 AAM, Cart. 23. Chanoine Paré et Mgr Bourget (Paris, mai 1856). 
cette Maison et en avoir essuyé un refus. Il faut de plus que le droit de l'évêque d'exiger d'elle $£ 1000$ ou $£ 2000$ soit bien clair. Autrement, on ne gagnerait qu'à ulcérer les esprits en pure perte. Si, pour vouloir aller plus vite que Dieu, on vient à créer dans le diocèse une funeste division, sous prétexte de bâtir un beau temple à la divine Majesté, on détruit au lieu d'édifier : et je redouterais, à coup sûr, beaucoup plus le désastre de cet incendie que celui du 8 juillet, dont nous allons bientôt faire le triste anniversaire. Savez-vous ce qui me préoccupe là-dessus plus que tout le reste ? Je vous le dis franchement; c'est que quand nous serons devant Dieu ce que nous devons être, les hommes cesseront d'être apathiques à notre égard. Ainsi notre sort est entre nos mains (....). Quoi qu'il en soit, je puis vous assurer que je suis bien décidé à mettre hache en bois en arrivant à Montréal, pour préparer les voies à cette reconstruction, et que je me sens, avec la grâce de Dieu, la ferme volonté de ne jamais lâcher prise jusqu'à ce que j'aie accompli cette œuvre, dont me charge la divine Providence ${ }^{39}$.

(à suivre)

LÉON POULIOT, s.j.

${ }^{39}$ AAM, Cart. 14, Mgr Bourget, Lettres pers., 1856-1859 (Paris, 18 juin 1856). 